Revista de Investigación Educativa 28

enero-junio, 2019 | ISSN 1870-5308 | Xalapa, Veracruz

Instituto de Investigaciones en Educación | Universidad Veracruzana

\title{
Comprensión lectora y medición de fluidez en universitarios de origen maya
}

\section{Reading comprehension and measurement of fluency in university students of mayan origin}

\author{
Eugenio Elías León-Islas ${ }^{\mathrm{a}}$ \\ Marisol May López ${ }^{\mathrm{b}}$ \\ Jorge Antonio Chi Tamayc
}

Recibido: 28 de agosto de 2018

Aceptado: 26 de diciembre de 2018

DOI: https://doi.org/10.25009/cpue.v0i28.2603

El objetivo de esta investigación fue analizar el nivel de comprensión de lectura y medición de fluidez en universitarios de origen maya. La muestra se conformó de 30 alumnos, 19 hombres y 11 mujeres. Del total, $36.7 \%$ es bilingüe y $63.3 \%$ únicamente habla castellano. La media en edad es 20.53 años. Todos cursan el primer semestre de universidad. El nivel de comprensión de lectura se mide mediante un texto de 699 palabras. Se utilizó el coeficiente de correlación lineal. Se obtuvo un coeficiente de $\mathrm{r}=.862$ entre la velocidad de comprensión lectora y la fluidez. Se obtuvo, además, un coeficiente de correlación de $r=-.864$, entre la fluidez en la lectura y el tiempo de lectura. El idioma de los sujetos no influye en el nivel de comprensión y fluidez. El género influye en el nivel de comprensión, pero no en la fluidez.

Palabras clave: Comprensión de lectura; comprensión de textos; estudiantes indígenas; estudiantes de primer ingreso; lectura.

a Maestro en Educación Intercultural. Profesor de asignatura, jefe de Departamento de bibliote-

ca, Universidad Intercultural Maya de Quintana Roo, México. 凶e eugenio.leon@uimaroo.edu.mx

b Licenciada en Lengua Inglesa y pasante de la maestría en Educación Intercultural. Analista

profesional, Universidad Intercultural Maya de Quintana Roo, México.

凶marisol.may@uimqroo.edu.mx

'Licenciado en Administración y pasante de la maestría en Administración. Analista profesio-

nal, Universidad Intercultural Maya de Quintana Roo, México. 凶 jorge.chi@uimqroo.edu.mx 
Eugenio Elías León-Islas, Marisol May

López, Jorge Antonio Chi Tamay

The objective of this research was to analyze the level of reading comprehension and fluency measurement in university students of Mayan origin. The sample consisted of 30 students, 19 men and 11 women. Of the total, $36.7 \%$ is bilingual and $63.3 \%$ only speaks Spanish. The average age is 20.53 years. All attend the first semester of university. The level of reading comprehension is measured by a text of 699 words. The linear correlation coefficient was used. A coefficient of $r=.862$ was obtained between the speed of reading comprehension and fluency. In addition, a correlation coefficient of $r=-864$ was obtained between reading fluency and reading time. The language of the subjects does not influence the level of comprehension and fluency. Gender influences the level of comprehension, but not fluency.

Keywords: Reading comprehension; texts comprehension; indigenous students; first-year students; reading.

\title{
$\approx$ \\ Comprensión lectora y medición de fluidez en universitarios de origen maya
}

\author{
Reading comprehension and measurement of fluency \\ in university students of mayan origin
}

\section{Introducción}

$\mathrm{Z}_{\text {gencia irrenunciable para el buen desempeño académico y una sólida formación }}^{\mathrm{n} \text { el }}$ profesional. En lo que va del siglo XXI, los resultados de las evaluaciones llevadas a cabo en materia de lectura de los estudiantes mexicanos por el Programa Internacional de Evaluación de Alumnos (PISA, por sus siglas en inglés) de la Organización para la Cooperación y Desarrollo Económicos (OCDE) no son nada halagadores.

Afirma Andere (2011) que de todos los miembros de la OCDE que han participado en las evaluaciones de PISA en los años 2000, 2003, 2006 y 2009, México ha sido 
consistente en ocupar el último lugar en todas las áreas evaluadas por este programa. Más aún en la evaluación llevada a cabo en 2009 con una población muestra de 38,250 estudiantes de 15 años de edad, un porcentaje considerable de estudiantes mexicanos se ubica en los niveles más bajos de desempeño de la competencia lectora (Castro, 2013). Adicionalmente a estos resultados, García (2017) escribe que en México se leen 2.9 libros al año. El bajo nivel de comprensión lectora y el reducido número de libros leídos por año en México parecen indicar que la lectura no ha cobrado gran relevancia ni en el ámbito social ni en el entorno ilustrado.

En el ámbito de la educación superior se escucha decir a muchos profesores que sus alumnos no leen, y si logran leer, su comprensión es muy baja. Frente a esta percepción, Carlino (2005) explica que leer y comprender textos académicos y científicos corresponde a culturas académicas que derivan de comunidades disciplinares diversas, donde los alumnos universitarios aún son forasteros.

La lectura es un proceso complejo (Yildiz \& Çetinkaya, 2017) puesto que involucra procesos psicológicos cognitivos, motivacionales, competencia léxica y dominio del código escrito. Miras, Solé y Castells (2013) mencionan que leer y escribir "se encuentran entre las tecnologías más poderosas de que disponemos para aprender y pensar" (p. 438). No obstante, Cassany (2010) puntualiza que el código escrito no es algo innato. Se adquiere. Los conocimientos lingüísticos provienen del exterior, son vastos y diversos. La lectura y la comprensión de textos, el estudio y la comprensión de las reglas gramaticales, la retención de fragmentos literarios son algunas de las actividades que ayudan a la adquisición del código escrito. Con todo, se hace hincapié en la importancia que tiene la comprensión lectora como una actividad cognitiva y habilidad lingüística necesaria para relacionarse de manera efectiva con la palabra escrita. Es más, la actividad didáctica y la lectura constante de textos académicos son los que resultan más efectivos para la adquisición del código escrito.

Por otro lado, PISA (OCDE, 2016, p. 146) define la lectura como "la capacidad de un individuo para comprender, emplear, reflexionar e interesarse en textos escritos con el fin de lograr metas propias, desarrollar conocimientos y su potencial personal, y participar en la sociedad". Es decir, que al leer no sólo se procesan visualmente las palabras y se identifican sus representaciones fonológicas, ortográficas, semánticas y lingüísticas, sino, además, se conectan las palabras utilizando las reglas de sintaxis para comprender el significado que subyace en cada oración de un texto escrito (Kendeou, McMaster \& Christ, 2016; Perfetti \& Stafura, 2014). Por consiguiente, toda oración expresa una acción, quién la efectúa y cuál es su objeto, en consecuen- 
cia, el sentido de cada oración comprende la interacción y construcción estrecha entre los sentidos de las palabras y las relaciones sintácticas que mantienen (Golder \& Gaonac'h, 2002).

La lectura es ampliamente reconocida, por varios investigadores, como una de las destrezas más importantes para el éxito académico (Schmitt, Jiang \& Grabe, 2011). En la lectura intervienen procesos de decodificación, recodificación y comprensión (Fajardo, Hernández \& González, 2012). Del mismo modo, se reconoce que es una actividad cognitiva que implica habilidades, estrategias, recursos de atención, aspectos motivacionales y competencia léxica (Shahnazari \& Dabaghi, 2014). De hecho, la lectura es una de las más trascendentales herramientas para el buen desempeño académico. Involucra la combinación de varias pericias y diferentes tipos de estrategias. Estas conllevan a relacionar personas, cosas y situaciones implicadas en un texto. Además, ayudan a determinar causas y efectos, a descubrir significados de palabras desconocidas en un escrito (Parodi, 2011).

En consecuencia, partimos del supuesto de que el nivel más profundo de la comprensión de un texto escrito resulta del propósito y los conocimientos previos que el lector tenga del tema, así como de la lectura atenta que realiza. Entonces, la lectura profunda implica la construcción de una representación mental coherente del texto en la memoria del lector y el uso apropiado de estrategias. Sin embargo, el éxito o la incapacidad de comprender, se ve influenciado por las características específicas del lector, las propiedades del texto y el contexto en el que tiene lugar la lectura (Britton \& Graesser, 1996; van Oostendorp \& Goldman, 1999). De igual forma, el logro del proceso de comprensión depende de la integración de los conocimientos previos de los lectores con la información textual (Goldman \& Bisanz, 2002; Kintsch, 1988, 1998; MacNamara, 2004; van den Broek, Virtue, Everson, Tzeng \& Sung, 2002). A su vez, el propósito se sustenta en la motivación sostenida y el propio interés, ya que estos son los impulsores de los lectores para seleccionar las estrategias adecuadas y realizar la lectura eficaz.

Desde esta perspectiva, la comprensión lectora es multidimensional (Kendeou et al., 2016) y está entre las más complejas capacidades y comportamientos humanos (Catts \& Kamhi, 2017). En consecuencia, la comprensión lectora consiste en la construcción de un modelo mental coherente del texto en la memoria de los lectores (Graesser, 2015).

En este marco de conceptos, las preguntas que nos planteamos son: ¿Cómo se relacionan la comprensión y la fluidez lectora en universitarios de ambos sexos? ¿El 
idioma influye en el nivel de comprensión y fluidez de textos escritos? ¿La comprensión y la fluidez lectora son iguales entre hombres y mujeres universitarios? ¿Qué nivel de comprensión tienen los universitarios, hombres y mujeres, al leer textos escritos?

Es difícil caracterizar con precisión a un alumno universitario bilingüe; para el presente estudio se concibe al alumno bilingüe como aquel que procede de una comunidad originaria donde la maya es la lengua predominante en el ámbito familiar y comunitario. Entonces, estos alumnos, si bien su lengua materna es la maya, logran ser personas bilingües al aprender a hablar en castellano durante su formación escolarizada preuniversitaria. En este contexto, las preguntas establecidas conllevan al propósito de esta investigación, que consiste en analizar y explicar la relación entre el nivel de comprensión lectora y la fluidez en estudiantes de origen maya, de ambos sexos, que cursan el primer semestre de universidad.

Yildirim, Rasinski y Kaya (2018) aseveran que la fluidez en la lectura y la comprensión se correlacionan de manera estrecha. Asimismo, la investigación ha demostrado que la fluidez, además de contribuir en la comprensión lectora, es un favorecedor importante en el éxito escolar y en el rendimiento general de la lectura (Lane et al., 2008).

Hudson, Pullen, Lane y Torgesen (2009) señalan que la fluidez de lectura es un constructo complejo y multifacético debido a la variabilidad en las definiciones. Sin embargo, sugieren que la fluidez de una persona al leer se asocia con la lectura competente, ya que además de ser precisa y eficiente implica una buena comprensión del significado del texto, aun cuando el tipo de texto sea diferente. Tal afirmación la sustentan en que la automaticidad a niveles subléxicos y léxicos es necesaria para una lectura fluida. Además, se conoce que los procesos automáticos ocurren tan rápido que están más allá del control o análisis consciente por parte del lector. En consecuencia, la fluidez en la lectura es la organización compleja de múltiples subprocesos que se activan en diferentes niveles, que van desde el reconocimiento de las letras y palabras hasta la construcción de significado. Por lo tanto, sin una lectura precisa de palabras, el lector no tendrá acceso al significado supuesto por el autor, y la lectura de palabras incorrecta puede llevar a interpretaciones erróneas del texto (Hudson, Lane \& Pullen, 2005).

En el presente estudio entendemos la fluidez de lectura como el reconocimiento preciso de las palabras y la rapidez con las que son leídas en un texto escrito en términos de un minuto. Por otra parte, aclaramos que de los alumnos universitarios 
que participaron en esta investigación algunos son bilingües, ya que hablan la lengua maya y el castellano, en tanto un segundo grupo habla únicamente castellano. Frente a esta distinción, se presume que, por ser estudiantes universitarios de origen maya, pertenecer a una región con alto rezago educativo y en muchos casos ser los primeros en ingresar a una institución de educación superior de corte intercultural, se incrementa la dificultad en lograr una lectura fluida y una comprensión profunda al leer textos escritos.

\section{Conceptos y dimensiones de la compresión}

La comprensión es una competencia transversal muy importante, no sólo para la lectura eficaz de textos escritos sino para otro tipo de actividades académicas. La comprensión es un proceso cognitivo complejo, necesario para todas las actividades cognitivas de alto nivel; incluye el aprendizaje, el razonamiento, la resolución de problemas y la toma de decisiones (McNamara \& Magliano, 2009). La comprensión, señala Perkins (1999), se presenta cuando la gente es capaz de pensar y actuar con flexibilidad a partir de lo que sabe. Los procesos de comprensión involucran conjuntos de resultados y realizaciones, tales como llevar a cabo análisis, elaborar juicios acabados, realizar síntesis y generar resultados que unan principios y conceptos clave para una disciplina. Por lo tanto, cualquier noción de comprensión, señala Gardner (1993) debe centrarse en las capacidades mostradas y en las operaciones realizadas de un ámbito disciplinar. Por consiguiente, el desempeño flexible es el resultado visible de la comprensión.

En realidad, la comprensión, como proceso cognitivo de gran complejidad, requiere la intervención activa de sistemas sensoriales y de diversos procesos inferenciales (Tijero, 2009). Aparte, la utilización de estrategias cognitivas y meta cognitivas deliberadas y conscientes son necesarias para lograr niveles más profundos de comprensión lectora (Graesser, 2007; McNamara, 2010); además, la enseñanza para la comprensión y las estrategias de lectura no siempre se desarrolla con acuciosidad y facilidad en el ámbito escolar. Tal parece que la enseñanza para la comprensión en el nivel de formación media superior es exigua e infrecuente (Wiske, 1999), lo cual repercute en la formación universitaria.

Los mecanismos de la comprensión lectora son diferentes y complejos. Se incluyen en estos los procesos cognitivos, los procesamientos léxicos, sintácticos, semánti- 
cos y pragmáticos (Golder \& Gaonac’h, 2002). Asimismo, la comprensión del significado de un texto encierra la construcción de una representación mental altamente coherente de la información del texto (King, 2007). Bajo estos supuestos, las intenciones de todo lector y la decodificación puntual de las proposiciones de un texto escrito son fundamentales para la comprensión efectiva de una lectura. No obstante, la comprensión de textos expositivos y científicos exige de los lectores la operación simultánea de varios niveles de abstracción (Alonso \& Mateos, 1985).

\subsection{Enfoques teóricos y modelos de la comprensión}

En torno a la comprensión lectora existen diversos modelos y enfoques teóricos (Alonso Tapia \& Mateos, 1985; Davoudi \& Hashemi Moghadam, 2015; Kendeou et al., 2016; McNamara \& Magliano, 2009; Shahnazari \& Dabaghi, 2014); si bien los enfoques teóricos y modelos coinciden en algunos puntos, se diferencian entre sí por el énfasis que ponen en algún punto clave. Algunos enfatizan la importancia de las habilidades lingüísticas, esto es, en el procesamiento fonológico, ortográfico y reconocimiento de palabras. Otros ponen mayor atención en los procesos relacionados con la construcción de la representación mental durante la lectura, es decir, en la dimensión psicolingüística, y algunos más se orientan a los resultados, el desempeño flexible, la acreditabilidad y comunicabilidad de lo comprendido (Parodi, 2011; Perkins, 1999). De igual forma se han desarrollado diversos dispositivos para medir y evaluar los diferentes niveles de comprensión (Fletcher, 2006).

La comprensión se considera comúnmente como el procesamiento de información para extraer significado (McNamara \& Magliano, 2009). Por consiguiente, en la comprensión lectora, el lector usa los conocimientos previos disciplinares y del mundo (McNamara, 2004). Además, recurre a los conocimientos sintácticos y semánticos para construir una imagen coherente del contenido del texto que lee (Kintsch, 1998; Kintsch \& Kintsch, 2005). Por ello, los conocimientos previos del individuo son componentes fundamentales para la comprensión en general, y de manera particular, muy importantes para leer un tipo específico de texto. En este sentido, Kintsch (1988) puntualiza que todos estos conocimientos son parte sustantiva para la comprensión efectiva de textos escritos.

La medición y la evaluación de la comprensión lectora son viables y factibles. Mansilla y Gardner (1999) reconocen la necesidad de hacer explícito un marco teórico que facilite la evaluación de los alumnos que logran una comprensión profunda. 
Estos autores examinan las cualidades de la comprensión en el contexto de la importancia que tiene para la enseñanza en diferentes ámbitos disciplinares. Consecuentemente, desarrollan una concepción teórica con dimensión práctica. Mansilla y Gardner (1999) sustentan la necesidad de desarrollar capacidades tales como el análisis crítico en operaciones complejas de cálculo, el uso práctico de la matemática y la solución de problemas a partir de la precisión de conocimientos disciplinares. Si bien en este proceso existen diferencias en el énfasis de cada meta y disciplina, la coincidencia consiste en que los alumnos que logran una comprensión profunda la reflejan a través de su desempeño.

\subsection{Niveles y dimensiones de la comprensión}

El marco conceptual que Mansilla y Gardner (1999) desarrollan para la comprensión, se estructura de cuatro dimensiones y cuatro niveles, los cuales, afirman, pueden distinguirse en el desempeño de los alumnos. Las cuatro dimensiones que estos autores proponen son: "el contenido, métodos, propósitos y formas de comunicación", y dentro de cada dimensión se describen cuatro niveles de comprensión: "ingenua, de principiante, de aprendiz y de maestro" (p. 230). Las dimensiones y los niveles de comprensión que formulan se tornan en una herramienta útil que facilita la sistematización de la evaluación de los desempeños virtuosos.

Desde otra perspectiva, la teoría sobre la comprensión lectora que desarrolla Parodi (2011) es de enfoque socio-psico-bio-lingüístico. Esta teoría concibe y postula el carácter multidimensional de la comprensión de textos escritos; en realidad, pone énfasis en la complejidad que encierra el macro proceso multidimensional de la comprensión y señala los diversos tipos de conocimientos que convergen.

La propuesta conceptual que desarrolla Parodi (2011) la denomina teoría de la comunicabilidad. La premisa fundamental radica en la concepción de que el ser humano posee una facultad innata del lenguaje y una conciencia de sí mismo. Estos rasgos prototípicos potencializan la capacidad para construir diversos significados de naturaleza simbólica en el proceso de lectura. El autor de esta teoría señala que en el marco de desarrollo de la conceptualización no ha dejado de poner atención y análisis en la revisión de diversas teorías y modelos de comprensión, con objeto de conocer el estado del arte y recuperar los aportes significativos.

La teoría de la comunicabilidad que concibe Parodi se sustenta en un principio básico y tres supuestos distintivos clave. El principio fundamental que rige su teoría 
(Parodi, 2011) es "la acreditabilidad de lo comprendido"; en tanto, las premisas principales que la sostienen, residen en el "supuesto de la cognición situada", el "supuesto de la interactividad", y el "supuesto de la socioconstructividad" (p. 148). De acuerdo con Parodi (2011) aquello que no se comprende en un texto escrito no se puede acreditar mediante la comunicación o explicación oral o escrita.

La presente investigación la sustentamos en la teoría de la comunicabilidad en tanto que asume la dimensión socio-psico-bio-lingüística y que la consideramos la más completa y acabada. En realidad, toda compresión lectora es producto de procesos mentales estratégicos, se construye en un contexto sociocultural y situacional determinado por la diversidad de textos y es un acto de comunicación interactiva.

Es común afirmar que todo lector es el resultado de una construcción progresiva de entornos sociales particulares, de una base biológica, de la conciencia de su yo, la automatización de diferentes procesos y el aprendizaje de diversas estrategias de lectura. Todo ello lleva a Parodi (2011) a afirmar que "la comprensión de textos escritos es un macro proceso multidimensional en cuyo núcleo convergen diversos tipos de información, de relaciones y de procesos y subprocesos" (p. 163). En consecuencia, la comprensión involucra un conjunto de conocimientos previos del propio lector y del mundo (McNamara, 2004), así como de la acción interactiva entre el lector, el texto y el autor del texto (Pérez, 2005). Ciertamente, la comprensión es un proceso cognitivo muy complejo, como el proceso de medición de la comprensión lectora también lo es (Fletcher, 2006). Sin embargo, se ha medido a través del tiempo por diferentes técnicas: entre las más conocidas y utilizadas se mencionan las de Opción múltiple, Preguntas verdadero-falso, Cuestionario, Preguntas abiertas, Recuerdo libre, Cloze o Resumen (Habib, 2016; Ibáñez, 2012; Pérez, 2005; Rupp, Ferne \& Choi, 2006).

\section{Niveles de procesamiento y comprensión de textos}

La comprensión de textos escritos con frecuencia se describe como el procesamiento en diferentes niveles: código de superficie, base del texto y modelo de situación (van Dijk \& Kintsch, 1992; Kintsch, 1998). En el área de la psicolingüística, MarmolejoRamos (2007) escribe que en torno al tema de la comprensión de la lectura se ha desarrollado un gran número de investigaciones por ser un asunto central de la cognición humana. Por consiguiente, se requiere la intervención científica del trabajo de psicólogos que provengan principalmente de la ciencia cognitiva. Esta ciencia explica 
la forma como el cerebro humano percibe y procesa la información; hace hincapié en que el cerebro necesita recursos experienciales y conceptuales para que la memoria mantenga la información procesada; también estudia las "operaciones inferenciales para integrar grupos de datos y procesos de construcción de conocimiento" (Marmolejo-Ramos, 2007, p. 332). Todo esto sucede en un marco interactivo entre lector y texto (Pérez, 2005).

Los primeros psicólogos cognitivos que sostuvieron que el sistema cognitivo construye representaciones estructuradas en varios niveles de codificación fueron Craik y Lockhart (1972). Estos autores afirman que los niveles de análisis y acopio de información crean trazas, huellas de memoria que contienen información codificada, referidas a distintos dominios cognitivos. Lockhart, Craik y Jacoby (1976) utilizan la noción de "dominios de procesamiento" para confirmar la idea de que el procesamiento visual de palabras, por ejemplo, pasa por etapas de análisis visual e impreso antes de someterse a los análisis articulatorios, etapas fonológicas, léxicas y conceptuales.

Particularmente, Craik y Lockhart (1972) sugieren que la persistencia de las trazas es una función de la profundidad del análisis; esto es, con niveles más profundos de análisis se asocian trazas más elaboradas, de mayor duración y más hondas. Es decir, no toda la información se almacena en un mismo formato, y de la misma manera, los hay para distintos aspectos de acuerdo con ciertas categorías de registro. En otras palabras, los niveles de análisis determinan las jerarquías de procesamiento. El procesamiento perceptivo es el nivel más superficial; en cambio, el análisis semántico corresponde al nivel de significado más profundo (Craik, 2002).

Sostienen Craik (2002) y Craik y Lockhart (1972) que el recordar, como actividad de la mente, es un procesamiento, y no son huellas de la memoria como entidades que deben ser buscadas, "encontradas" y reactivadas. Es decir, las operaciones de codificación que realiza la memoria se deben conceptualizar como procesos subyacentes a la percepción y la comprensión, y que la recuperación se torna en el corolario de la codificación. En consecuencia, recordar refleja los tipos cualitativos de análisis que se han realizado durante los procesos de codificación inicial, de percepción y comprensión, y que el procesamiento más profundo se asocia con niveles más altos de evocación posterior. Sin embargo, el procesamiento más profundo no se reduce a una extensión o prolongación del procesamiento superficial, sino a la comprensión decodificada del texto leído.

El tiempo ha transcurrido y la noción de niveles de procesamiento, desde la psicología cognitiva, que plantearon Craik y Lockhart (1972), ha evolucionado. Ahora, 
por lo general, se investiga y escribe sobre niveles de coherencia y cohesión textual (van Dijk, 1980; van Dijk \& Kintsch, 1992). Asimismo, se inquiere sobre los niveles de comprensión de textos. Los niveles reconocidos con más frecuencia se conocen como el código de superficie, el texto base proposicional, la micro estructura, la macro estructura y el modelo de situación referencial. Estos describen los diferentes tipos y niveles de información que pueden presentarse en la medida que ocurren los procesos de comprensión (Kintsch \& Rawson, 2005).

En este sentido, los resultados de las investigaciones sobre la comprensión lectora confirman la existencia de diferentes niveles de comprensión (Snow, 2010). Este autor proporciona una taxonomía de cuatro niveles. El primero de estos concibe una comprensión básica. Se trata de una comprensión literal, es decir, hace referencia, únicamente, al reconocimiento del significado de las palabras de un texto escrito. El segundo nivel implica la capacidad de construir una representación mental de las ideas presentadas textualmente (Kintsch, 1998; Kintsch \& Kintsch, 2005); en este nivel ya no sólo es reconocer y comprender las palabras. En otros términos, la comprensión lectora consiste en la construcción de representaciones situacionales y referenciales. El tercer nivel conlleva una comprensión más elaborada, una comprensión profunda del texto; corresponde a la formulación de preguntas y obtención de respuestas que se derivan de la lectura de un texto, busca establecer relaciones entre éste y otros, entre causas y efectos; se dirige a identificar la postura del autor del texto, incluso a criticar el argumento expuesto en él, se realizan inferencias.

Desde esta taxonomía, señala Snow (2010) que el nivel de mayor profundidad es aquel que involucra procesos de comprensión altamente elaborados. En consecuencia, para lograr este nivel se requiere el manejo de conceptos de estudios disciplinarios, estrategias de lectura, elaboración de inferencias y el aprendizaje profundo a partir del texto. Los procesos de comprensión en este nivel sólo se pueden esperar de aquellos lectores que cultivan un campo disciplinar y lo dominan. Este nivel de comprensión de lectura diferencia a los buenos de los malos lectores.

No obstante, Pearson y Hamm (2005) agregan, citando a Johnston (1984), que la comprehensión, o "comprensión", por su propia naturaleza, es un fenómeno que sólo se puede evaluar, examinar y observar indirectamente. Por lo general, las personas dicen aquello que entendieron, que les desconcertó, que disfrutaron o les molestó de un texto. O comúnmente se les cuestiona de algún modo sobre "el texto", requiriéndoles que recuerden su esencia, la idea principal o sus detalles principales, 
haciéndoles preguntas específicas sobre su contenido y propósito e insistiendo en una interpretación y crítica de su mensaje.

Sin embargo, los modelos de comprensión de lectura y el cómo evaluarlos han evolucionado a lo largo del siglo XX. Al respecto, Pearson y Hamm (2005) refieren que los primeros acercamientos sobre la evaluación de la lectura fueron proporcionados por Kelly y Thorndike en 1916 y 1917, respectivamente. Sin duda cada aportación teórica en torno al procesamiento de lectura y los niveles de comprensión es valiosa e importante.

El enfoque que asumimos, en cuanto a los niveles de procesamiento y comprensión de textos escritos, es el que desarrolla y sostiene Kintsch (1988; 1998), el Modelo de Construcción-Integración (C-I). La teoría que subyace en el modelo C-I establece que el producto de la comprensión del lenguaje resulta de una representación de aquello a lo que el lenguaje se refiere y no al lenguaje en sí mismo. El modelo de Kintsch distingue tres niveles de procesamiento en la comprensión del texto: estructura de superficie, la base del texto o representación proposicional y la construcción de la situación o modelo del mundo. Este último es el nivel más profundo en cuanto que integra la información del texto con el conocimiento del mundo del lector y hace referencia al mundo único que el texto informa.

En consecuencia, al asumir la comprensión lectora como un proceso de interacción entre el texto y el lector, se reconocen como importantes, al menos tres niveles de comprensión lectora: la literal, la inferencial y el nivel de comprensión crítico. En este sentido, el logro de la comprensión lectora es aprender a entender la escritura como un proceso de interacción entre el lector y el escritor de manera contextual (Perfetti, Landi \& Oakhill, 2005).

\section{Evaluación y medición de la comprensión lectora}

La investigación del proceso de comprensión de textos ha provocado en este campo, a través del tiempo, una gran efervescencia entre la comunidad de investigadores debido al impacto que tiene en el desempeño académico y humano (Pearson \& Cervetti, 2017).

En los más de 40 años de investigación en la teoría de la comprensión de la lectura, el proceso de instrucción de cómo leer, de las formas para llevar a cabo la evaluación y de la elaboración de currículo dan cuenta de ello (Pearson \& Cervetti, 2017). 
Después de más de cuatro décadas de investigación sobre la comprensión de la lectura, se ha llegado a la conclusión de que la comprensión, por su propia naturaleza, es un fenómeno que sólo se puede observar indirectamente (Pearson \& Hamm, 2005). La comprensión, expresa Cassany (2010), se construye en la mente de cada lector que vive en contextos diversos y posee disímiles experiencias de la realidad. Esto es un hecho innegable.

Sin duda, la mayoría de las personas y un gran número de alumnos universitarios dicen aquello que entendieron, aquello que les sorprendió, que disfrutaron, o expresan aquello que les molestó al realizar la lectura de un texto que se les encomendó leer. Asimismo, los profesores preguntan a sus alumnos sobre el texto leído; les requieren, principalmente, que recuerden las ideas principales, la esencia, el contexto o los detalles importantes del texto. Les hacen preguntas específicas sobre su contenido y propósito o insisten en que hagan una interpretación y crítica del mensaje del autor. Sin embargo, se conoce que las escuelas, entre sus tareas principales, tienen que asignar lecturas a los alumnos y exigir que comprendan los textos que se les fija. De igual forma, los maestros son quienes promueven y evalúan la comprensión de la lectura que realizan los alumnos. La evaluación, a su vez, es una práctica pedagógica ineludible en el proceso de enseñanza aprendizaje. Pero usualmente se utiliza para reorientar las actividades de enseñanza y reconocer el grado de aprendizaje de los alumnos (Habib, 2016), no obstante, poco se percatan de las habilidades y estrategias que los alumnos tienen para comprender un texto escrito.

En la presente investigación estamos de acuerdo en que la evaluación de la lectura es una tarea compleja (Rupp, Ferne \& Choi, 2006) y colmada de dificultades debido al conjunto de factores que intervienen en el acto lector. Los presupuestos teóricos en torno a la evaluación indican que a la hora de plantear una se han de tener en cuenta los indicadores más sensibles para reflejar el grado de comprensión que tiene el lector. Sin embargo, la evaluación de la lectura se ha centrado en pruebas de velocidad y comprensión; se mide esta comprensión en diferentes formas, bien por medio de recuerdo libre, por preguntas de verdadero o falso o por preguntas de alternativa múltiple (Fletcher, 2006); aun así, no deja de ser compleja dicha evaluación.

Kerlinger y Lee (2002) indican que la medición es una de las piedras angulares de la investigación. La mayoría de los métodos de recolección de datos que eventualmente requieren algún tipo de cuantificación se basa en la medición. El constructo “medición” lo conceptualiza muy bien Stevens (1968) y lo define como "la asignación 
de valores numéricos a objetos o eventos, de acuerdo con ciertas reglas" (p. 854). Esta definición, sin duda, enuncia la esencia de la medición. Da pie a la posibilidad teórica de medir cualquier cosa, siempre y cuando las reglas que se apliquen a la medición se sustenten de manera racional o empírica (Stevens, 1946). Por consiguiente, los números que derivan de la medición son resultado de una serie de condiciones que contienen la huella de las operaciones por las que se obtuvieron (Stevens, 1968). Sin embargo, el hecho de que los numerales se puedan asignar bajo diferentes reglas conduce a diferentes tipos de escalas y diferentes tipos de medidas. Los tipos de escalas comunes y utilizadas para la asignación de numerales son suficientemente conocidas. La escala nominal, la ordinal, la de intervalo y la razón son utilizados en la investigación científica (Stevens, 1968).

Con base en el concepto medición propuesto por Stevens (1946) y que aquí se retoma, asumimos que la lectura es una conducta observable y en consecuencia, medible, cuantificable, factible de asignarle numerales. En suma, es posible medir el tiempo dedicado a una lectura, incluso medir el número de palabras leídas con rapidez y precisión, igual que la velocidad de su comprensión.

En un estudio llevado a cabo en 2011 con 4,351 estudiantes de ambos sexos, de 11 Instituciones de Educación Superior (IES), que recién ingresan a las IES del área metropolitana de la ciudad de México, se reporta que los mejores entre los que aplican a las convocatorias alcanzan 5.9\% en comprensión de la lectura en la escala de 1-10. En el mismo estudio se indica que un alumno estándar de recién egreso del nivel de secundaria lee 155 palabras por minuto; a partir de este puntaje se calculó que un alumno egresado de bachillerato con un nivel estándar no le sería difícil leer 170 palabras por minuto (Sánchez, 2014).

La medición, en consecuencia, es esencial para determinar las relaciones funcionales, para el descubrimiento del orden y la regularidad de las características o propiedades que se buscan medir. En este sentido, los autores de esta investigación, apegados a los criterios de medición, conformaron una muestra de 30 alumnos del primer semestre de universidad, que cursaban la asignatura denominada "Taller de Lectura Analítico Crítica y Comprensión de Textos”, para describir y analizar el nivel de fluidez, la velocidad de comprensión de lectura, el tiempo de lectura y el grado de comprensión lectora. 


\section{Método}

\subsection{Participantes}

En la presente investigación participa una muestra de 30 alumnos universitarios que cursan el primer semestre en la Universidad Intercultural Maya de Quintana Roo (UIMQROO). De esta muestra de alumnos, $36.7 \%$ corresponde al sexo femenino y $63.3 \%$ al sexo masculino. En cuanto al idioma, $36.7 \%$ son bilingües, puesto que hablan maya y castellano; en tanto que 63.3\% únicamente habla el castellano. La edad promedio de los participantes en la investigación es de 20.53 años. Con relación al origen de procedencia se observa que $43.33 \%$ proviene de comunidades rurales y $56.66 \%$ de la zona urbana. Los alumnos que participan en esta investigación cursan la asignatura: “Taller de Lectura Analítico Crítica y Comprensión de Textos”.

\subsection{Variables $e$ instrumento}

Para la medición del nivel de comprensión y fluidez de lectura de cada uno de los 30 participantes en esta investigación se utilizó como instrumento una computadora móvil en donde se instaló un software denominado: Lectura rápida y comprensión (Siglo XII). Este software fue elaborado por un equipo multidisciplinario de profesionales conformado por lingüistas, psicólogos cognitivos, informáticos y docentes de la lengua castellana. Entre los objetivos principales está diagnosticar el nivel de comprensión lectora y facilitar ejercicios de diferente complejidad para el desarrollo de la velocidad en la lectura y la comprensión de textos escritos, pretendiendo disminuir el tiempo de lectura y elevando el nivel de comprensión.

El texto que sirvió de prueba lectora fue un extracto del capítulo 1 de la novela histórica: La isla bajo el mar de la escritora Isabel Allende. La autora de la novela narra, en el extracto denominado Zarité, la vida de una esclava a quien le gusta disfrutar la música y a través de ésta encuentra la libertad que no tenía. El tema que aborda es la esclavitud. El relato es de un estilo ágil, metafórico, bien estructurado, no cansa al leerlo. Además, la novelista es ampliamente conocida en el medio literario de habla hispana y en el entorno educativo medio superior.

Al terminar de leer el relato, el software formula 10 preguntas de opción múltiple cuyo valor de respuesta es equivalente a 10, 5 y o puntos. Si la respuesta es correcta el lector obtiene una puntuación de 10 puntos, en caso de ser incorrecta es de o puntos 
o de 5 si se aproxima a la respuesta correcta. Algunas de las preguntas planteadas para medir el nivel de comprensión y fluidez son: ¿Cuáles son los dos grandes temas que Zarité platica en su relato? ¿Cuál es el tema principal de la lectura? ¿Qué significado encierra la frase "esclavo que baila es libre... mientras baila"? ¿Qué es para Zarité la música? Finalmente, el alumno lector recibe del software una puntuación en los siguientes cuatro aspectos: Tiempo de lectura, porcentaje de comprensión lectora, velocidad de palabras por minuto y velocidad de comprensión lectora.

Al disponer del instrumento y comprobar que efectivamente mide cuatro variables clave: tiempo de lectura, porcentaje de comprensión de lectura, además de medir la velocidad de palabras en un tiempo determinado y la velocidad de comprensión lectora, se decidió utilizar dicho software considerando las variables sexo e idioma de los sujetos.

\subsection{Procedimiento}

Para realizar la medición de la comprensión y fluidez de la lectura, se pide a dos profesores y a una profesora de la UIMQROO, que imparten en el área de Formación básica la asignatura: "Taller de Lectura Analítico Crítica y Comprensión de Textos”, que permitan la participación del 10\% de sus alumnos para leer un texto narrativo, escrito en dos párrafos de un total de 699 palabras, con el fin de medir el nivel de comprensión y fluidez lectora.

Cada alumno lee de manera individual la lectura Zarité, siguiendo cada paso que le indica el software instalado en una computadora móvil hasta completar el ciclo, es decir, lectura de los dos párrafos y respuesta a cada una de las diez preguntas. Finalmente, el software arroja los resultados en forma resumida; estos resultados son registrados en un formato que contiene las cuatro variables: tiempo de lectura, porcentaje de comprensión, velocidad de palabras por minuto y velocidad de comprensión.

\section{Resultados}

\subsection{Medición de las variables de la conducta lectora según el idioma}

Los datos descriptivos que se obtienen de cada uno de los participantes de la muestra, después de leer el texto de 699 palabras, son procesados mediante el software estadístico denominado Statistical Package for the Social Science (SPSS) versión 22. 
En la Tabla 1, se observa la distribución de la muestra de alumnos universitarios que participan en la investigación, cursan el primer semestre de universidad y están inscritos en la asignatura: "Taller de Lectura Analítico Crítica y Comprensión de Textos”. Se observa el número de alumnas y alumnos distribuidos según el género y el idioma que hablan. Se hace notar que tanto las alumnas como los alumnos que hablan la lengua maya también entienden y hablan el castellano.

\section{Tabla 1. Distribución de sujetos según el idioma y el género}

\begin{tabular}{lccc}
\hline \multirow{2}{*}{ Lengua } & \multicolumn{3}{c}{ Género } \\
\cline { 2 - 4 } & Femenino & Masculino & Total \\
\hline Bilingüe (Maya-Castellano) & 4 & 7 & 11 \\
\hline Castellano & 7 & 12 & 19 \\
\hline Total & 11 & 19 & 30 \\
\hline
\end{tabular}

Fuente: Resultados de los datos sociodemográficos.

En la Tabla 2 se pueden observar los resultados promedio de cada variable con respecto al idioma. El promedio en las cuatro variables difiere. Sin embargo, llama la atención el promedio que se da entre el grupo que únicamente habla castellano y el grupo bilingüe en lo que respecta a la fluidez de palabras por minuto. La diferencia es de 16.59; no obstante, la diferencia entre las medias no es significativa ya que al aplicar la ANOva de un factor se obtuvo un valor de $\mathrm{F}=2.496$, el cual es menor a 4.1830 al nivel de significancia de 0.05 . Con estos datos no se puede afirmar que la magnitud de diferencia sea significativa.

Pero si se compara la media de la variable porcentaje de comprensión lectora con el promedio de fluidez, del grupo bilingüe y del grupo que habla castellano, entonces se puede decir que el idioma no influye en el nivel de comprensión lectora. 


\section{Tabla 2. Comparación de grupos al $95 \%$ de intervalo de confianza para la media}

\begin{tabular}{lllcccc}
\hline \multicolumn{1}{c}{ Variables } & \multicolumn{1}{c}{ Idioma } & N & Media & $\begin{array}{c}\text { Error } \\
\text { estándar }\end{array}$ & DS & Varianza \\
\hline \multirow{2}{*}{ Tiempo de lectura } & Castellano & 19 & 4.58 & .258 & 1.124 & 1.263 \\
\cline { 2 - 7 } & Bilingüe & 11 & 5.45 & .455 & 1.508 & 2.273 \\
\hline \multirow{2}{*}{\begin{tabular}{l} 
\% de comprensión lectora \\
\cline { 2 - 7 }
\end{tabular}} & Castellano & 19 & 66.58 & 2.327 & 10.145 & 102.924 \\
\cline { 2 - 7 } $\begin{array}{l}\text { Fluidez de palabras por } \\
\text { minuto }\end{array}$ & Bilingüe & 11 & 68.18 & 3.389 & 11.241 & 126.364 \\
\hline Castellano & 19 & 147.50 & 11.311 & 49.302 & 2430.66 \\
\hline $\begin{array}{l}\text { Velocidad de comprensión } \\
\text { lectora }\end{array}$ & Bilingüe & 11 & 130.91 & 9.475 & 31.424 & 987.491 \\
\cline { 2 - 7 } & Castellano & 19 & 103.21 & 6.462 & 28.167 & 793.398 \\
\hline
\end{tabular}

Fuente: Resultados estadísticos descriptivos procesados en SPSS v.22.

Debido a este resultado se aplica el estadístico coeficiente de correlación lineal de Pearson para determinar el grado de estrechez de estas variables con el idioma de los sujetos, puesto que los promedios del tiempo de lectura y porcentaje de comprensión lectora no varían demasiado. También se aplica la correlación lineal con las restantes variables para determinar el grado de estrechez entre ellas.

\subsection{Medición de las variables de la conducta lectora según el género}

El género de los sujetos de la muestra del presente estudio es otra de las variables que se buscó analizar con relación al porcentaje de comprensión de lectura, fluidez de palabras por minuto, velocidad de comprensión lectora y el tiempo de lectura.

Los resultados de las investigaciones señalan que, al leer las palabras, oraciones y frases con precisión y rapidez, la comprensión lectora se incrementa en general. Sin embargo, no hay estudios que indiquen que la variable género influya en ambas variables. Los resultados estadísticos descriptivos se pueden observar en la Tabla 3 . En esta tabla, al observar los promedios y la varianza obtenidos entre las variables independientes y las variables en estudio, se detectan ciertas diferencias. Por ejemplo, el promedio de comprensión lectora del grupo de alumnos es menor que el de las mujeres. Sin embargo, en lo que compete a la fluidez, el promedio de los alumnos es mayor 
que el de las alumnas. Es decir, el grupo de alumnas obtiene un porcentaje mayor de comprensión que los alumnos a pesar de baja fluidez.

\section{Tabla 3. Comparación de grupos al $95 \%$ de intervalo de confianza para la media}

\begin{tabular}{lcccccc}
\hline \multicolumn{1}{c}{ Variable } & Género & N & Media & $\begin{array}{c}\text { Error } \\
\text { estándar }\end{array}$ & DS & Varianza \\
\hline \multirow{2}{*}{ Tiempo de lectura } & Femenino & 11 & 5.09 & .368 & 1.221 & 1.491 \\
\cline { 2 - 7 } & Masculino & 19 & 4.74 & .323 & 1.408 & 1.982 \\
\hline \multirow{2}{*}{\begin{tabular}{l} 
F de comprensión lectora \\
\cline { 2 - 7 }
\end{tabular}} & Memenino & 11 & 73.64 & 3.240 & 10.745 & 115.455 \\
\cline { 2 - 7 } $\begin{array}{l}\text { Mluidez de palabras por } \\
\text { minuto }\end{array}$ & Femenino & 19 & 63.42 & 1.914 & 8.342 & 69.591 \\
\cline { 2 - 7 } & Masculino & 19 & 154.21 & 12.155 & 52.982 & 2807.064 \\
\hline $\begin{array}{l}\text { Velocidad de comprensión } \\
\text { lectora }\end{array}$ & Femenino & 11 & 99.27 & 5.552 & 18.412 & 339.018 \\
\cline { 2 - 7 } & Masculino & 19 & 97.42 & 7.431 & 32.389 & 1049.035 \\
\hline
\end{tabular}

Fuente: Resultados estadísticos descriptivos procesados en SPSS v.22.

De acuerdo con estos datos se observa que el promedio obtenido por los alumnos en lo que compete a fluidez de palabras por minuto es superior al promedio obtenido por las alumnas; no obstante, el porcentaje de comprensión de las alumnas es ligeramente superior al porcentaje de comprensión de lectura de los alumnos. Para corroborar y verificar si las diferencias de estos promedios son significativas se aplicó el estadístico ANOVA.

El resultado que se obtuvo del análisis de varianza de un factor es de $\mathrm{F}=8.456$, el cual es mayor al requerido al nivel de significancia de 0.05 . Con este dato se afirma que la magnitud de diferencia es significativa para la variable género y el nivel de comprensión, pero no en la variable fluidez. A partir de este resultado se confirma que el grupo de alumnos universitarios de la muestra comprende menos al leer que el grupo de las estudiantes universitarias.

\subsection{Método estadístico de inferencia y porcentaje de comprensión lectora}

Frente a los resultados descriptivos y ante las preguntas que guiaron esta investigación, se aplicó el estadístico coeficiente de correlación de Pearson con el propósito de 
conocer si existe alguna correlación positiva o negativa entre la velocidad de palabras por minuto (VPM) y el porcentaje de comprensión lectora (\%CL). Asimismo, se buscó determinar la existencia de asociación estrecha u holgada entre la VPM y la velocidad de comprensión lectora (VCL). Los resultados del coeficiente de correlación lineal de Pearson se muestran en los siguientes diagramas de dispersión.

La Figura 1 muestra la dispersión de resultados de datos de la variable (X) VPM y la variable $(\mathrm{Y}) \% \mathrm{CL}$. Asimismo indica el grado de relación entre una y otra variable.

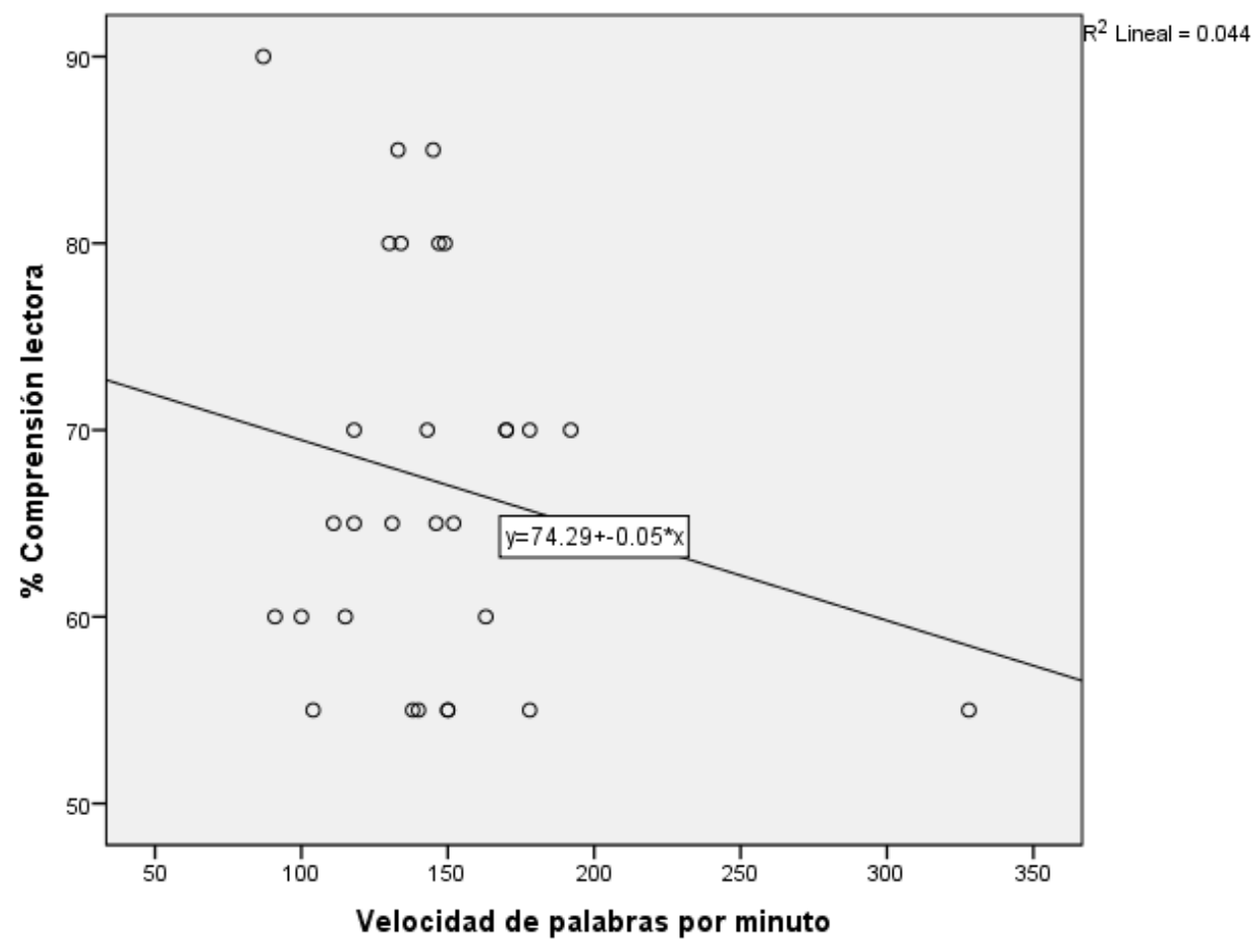

Figura 1. Fluidez de palabras por minuto y porcentaje de comprensión

Fuente: Figura elaborada en SPSs v.22 con datos de lectura.

Se considera que la velocidad de palabras por minuto se asocia con el tiempo de lectura, es decir, entre mayor número de palabras se lean, el tiempo de lectura aumenta y la comprensión textual también. Bajo estos supuestos, los resultados se expresan en las siguientes figuras. 
El gráfico de dispersión de la Figura 1, muestra una correlación lineal con valor de $r=.044$, este valor indica que no existe correlación, es decir, a mayor número de palabras leídas no garantiza el incremento de porcentaje de comprensión de un texto. No obstante, este resultado tampoco significa que para tener un porcentaje alto se deba leer a baja fluidez de lectura por minuto.

No así la Figura 2; en ésta se observa una correlación significativa en el nivel de 0.01 con un valor de $r=.86$, entre la variable velocidad de palabras por minuto y la variable dependiente comprensión lectora. Se puede concluir que en la medida que el lector aumenta el número de palabras leídas, también se incrementa la velocidad de comprensión de un texto escrito.

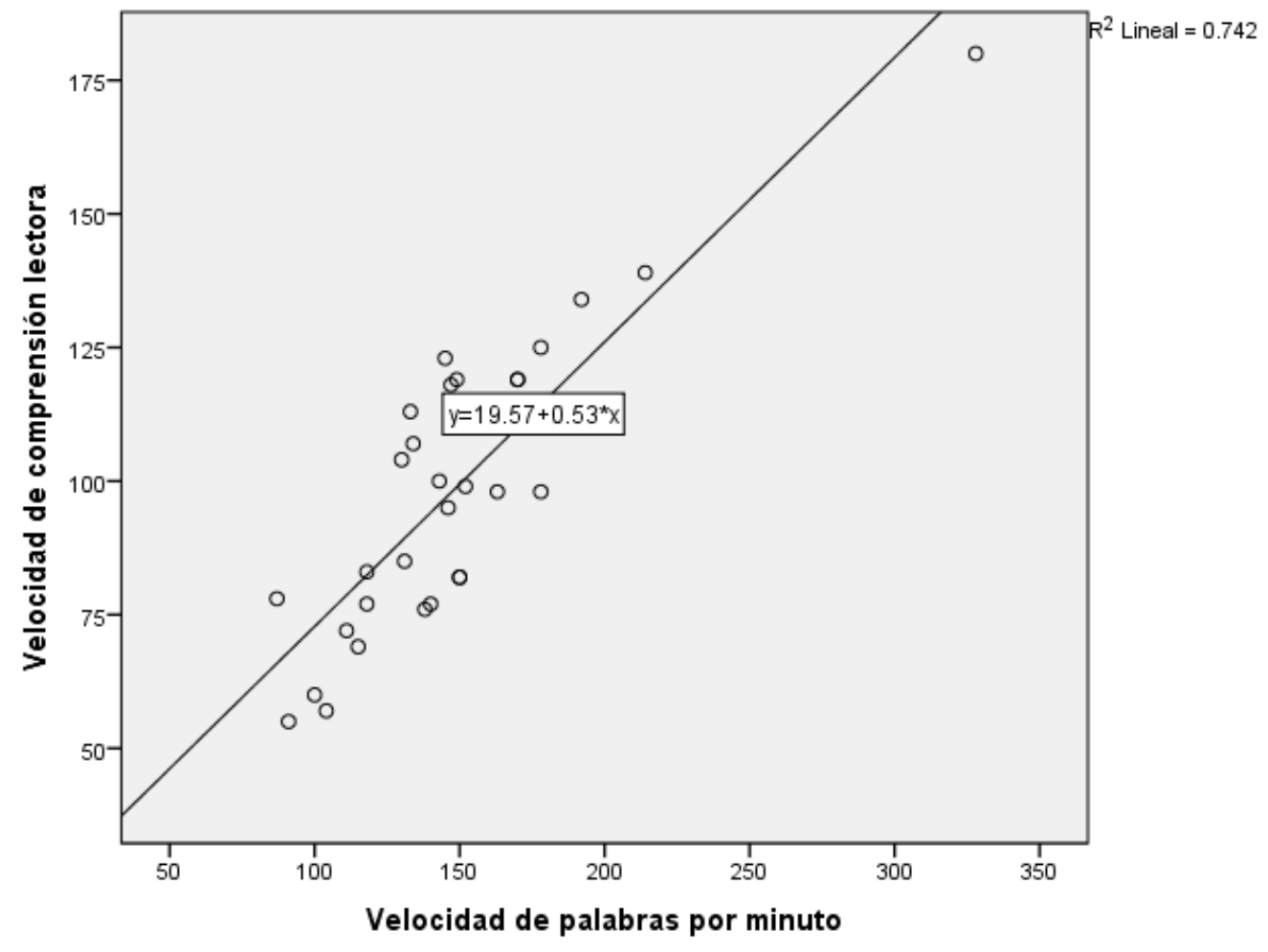

Figura 2. Relación entre velocidad de palabras por minuto y velocidad de comprensión

Fuente: Figura elaborada en SPSS v.22 con datos de lectura. 
Sin embargo, el diagrama de dispersión de la Figura 3 muestra una correlación negativa significativa en el nivel de 0.01 con un valor de $r=-.82$. Este resultado indica que en la medida que se invierte más tiempo en la lectura de un texto, disminuye de manera proporcional la velocidad de comprensión del texto escrito.

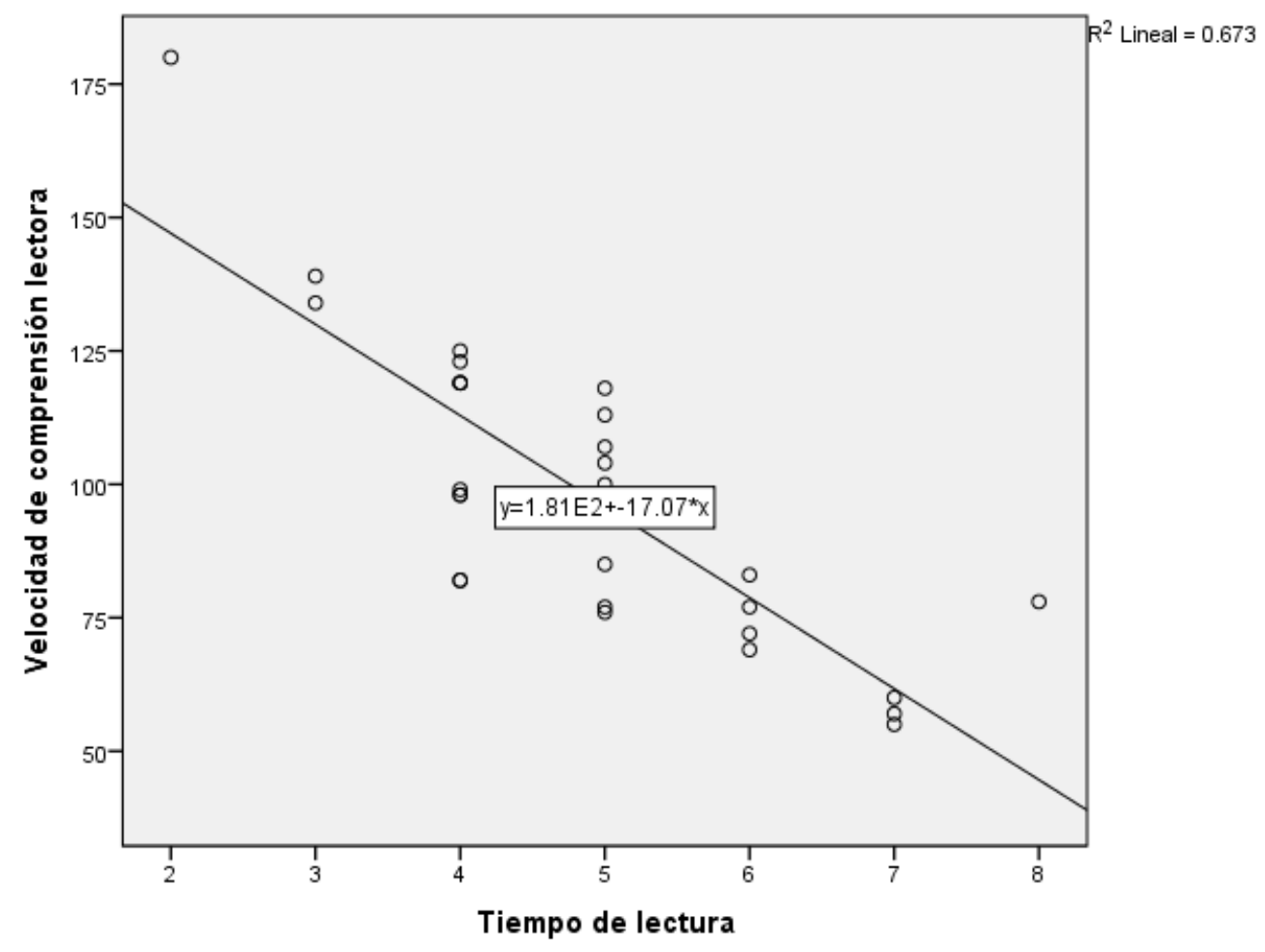

Figura 3. Relación entre tiempo de lectura y velocidad de comprensión

Fuente: Figura elaborada en SPSs v.22 con datos de lectura.

En el mismo sentido, se puede observar la conducta del lector en la gráfica de dispersión de la Figura 4, que indica que la velocidad de palabras leídas por minuto se correlaciona con el tiempo de lectura de manera significativa en el nivel de 0.01 pero de manera negativa, es decir con un valor de $r=-.86$. 


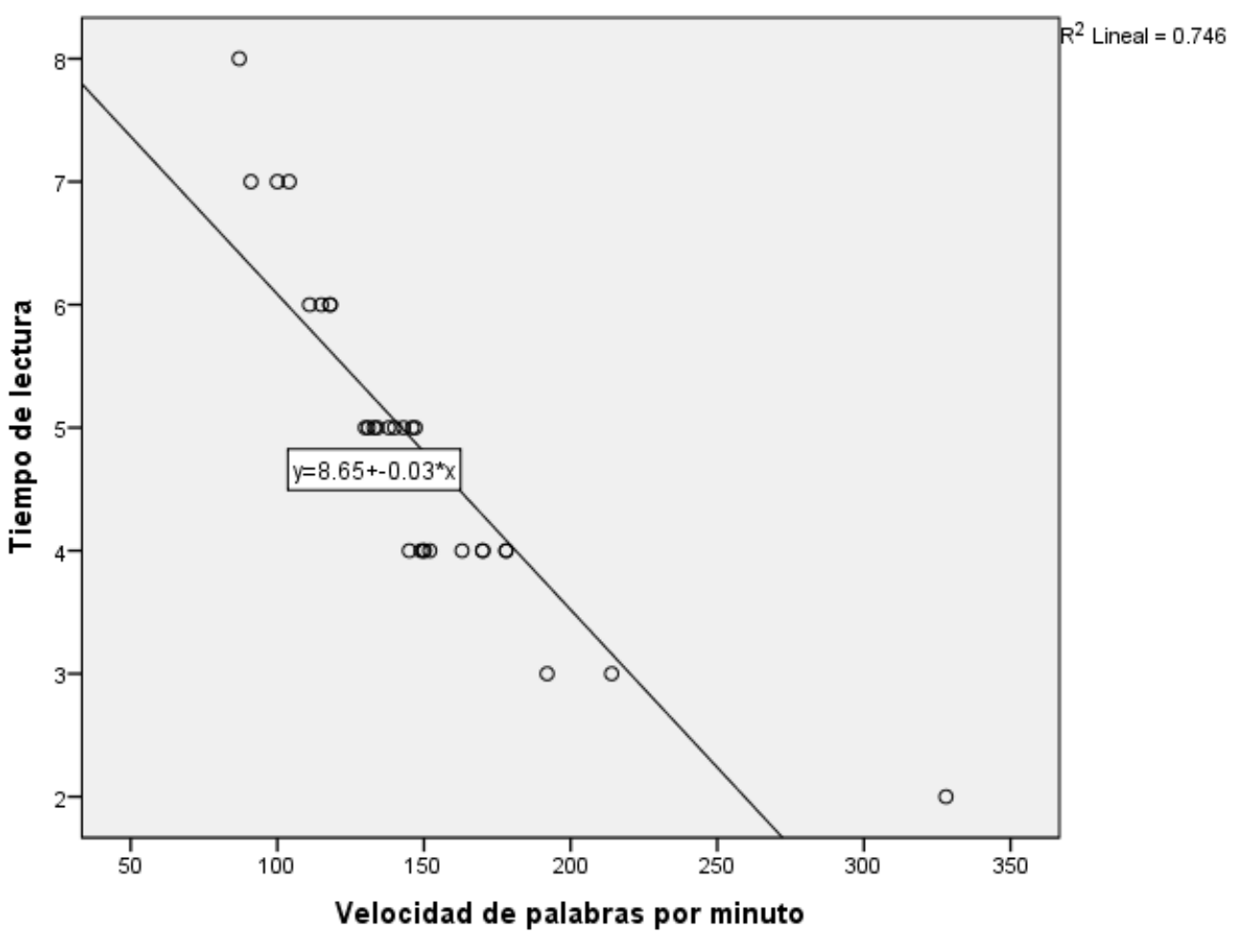

Figura 4. Relación entre velocidad de palabras por minuto y tiempo de lectura

Fuente: Figura elaborada en SPSs v.22 con datos de lectura.

Este resultado señala que en la medida que aumenta el tiempo de lectura, la velocidad de palabras también se reduce.

\section{Conclusiones}

En la presente investigación se propuso analizar y explicar la relación del nivel de comprensión y fluidez de lectura considerando las variables idioma y sexo en estudiantes universitarios de origen maya que cursan el taller de "Lectura Analítico Crítica y Comprensión de Textos”. Se realizaron dos tipos de análisis estadísticos, uno 
descriptivo y el segundo inferencial, a fin de medir y explicar el nivel de comprensión, la fluidez de lectura de textos y la correlación entre estas variables.

Mediante la aplicación de estadísticos descriptivos se determinó el promedio de tiempo de lectura, éste fue de 4:87, cuatro minutos con ochenta y siete milisegundos. El porcentaje de comprensión resultó de $67.17 \%$. La fluidez de lectura, esto es, la precisión y rapidez de lectura de palabras por minuto que fue de 147.50. Asimismo, se realiza el análisis del coeficiente de correlación lineal de Pearson, cuyos resultados se exponen en las gráficas de dispersión (Figuras 1 a 4).

Los resultados del análisis de correlación indican que hay una relación positiva entre la fluidez de lectura y la velocidad de comprensión. Estos resultados se corroboran con las investigaciones que sugieren que la fluidez de lectura de palabras por minuto y la comprensión de textos se asocian a nivel de palabras y oraciones $(\mathrm{Fu}-$ magalli, Barreyro \& Jaichenco, 2017; Yildirim, Rasinski \& Kaya, 2017), así como los niveles más altos de velocidad de comprensión lectora se asocian con los niveles más altos de fluidez de palabras. Este resultado se confirma con los resultados de la investigación de Yildirim, Rasinski y Kaya (2018) y de Jenkins, Fuchs, Van den Broek, Espin y Deno (2003). Asimismo, esta correlación no depende de los tipos de texto y género (Rasinski, Reutzel, Chard \& Linan-Thomson, como se citó en Yildirim et al., 2017).

Tal parece que ser bilingüe, es decir, hablar maya y castellano, no influye en el tiempo de lectura de un texto escrito, ni en la fluidez y la velocidad de comprensión de palabras por minuto, como se corrobora por los resultados del análisis de varianza. Esta conclusión se deduce de los resultados que se observan en la Tabla 2 donde se muestra el promedio de cada una de las variables consideradas y relacionadas con el idioma de los sujetos en estudio y se observa las diferencias del promedio. En la misma tabla, si bien se constata cierta diferencia en el promedio, en lo que compete a porcentaje de comprensión y fluidez lectora, no se puede afirmar que la magnitud de diferencia sea significativa. Posiblemente el número de sujetos de la muestra de la investigación fue insuficiente para llegar a una conclusión confiable.

En lo que corresponde a la variable género de los sujetos se concluye que interfiere en el grado de fluidez, en el tiempo de lectura, pero no en el nivel de velocidad de comprensión de textos, como se puede observar en la Tabla 3. En consecuencia, se deduce que los niveles bajos en el grado de fluidez y tiempo de lectura son resultado de la carencia de instrucción en las estrategias y la fluidez de comprensión, que consideramos se han descuidado en los niveles de estudio preuniversitario. La 
exploración de literatura que relaciona la fluidez de la lectura y la comprensión lectora señala que varios son los estudios empíricos que informan que hay correlaciones positivas de moderadas a altas entre la fluidez de la lectura y la comprensión lectora (Kim, Wagner \& Lopez, 2012).

La lectura juega un papel importante en los tres niveles del sistema educativo mexicano. La mejora de las habilidades de lectura es de vital importancia para un buen desempeño académico, sólo se puede lograr a través de la instrucción apropiada de estrategias efectivas de lectura (Ulker, 2017). Por tanto, la comprensión lectora depende directamente de las estrategias que utilizan los lectores (Bachman \& Palmer, 2009; Blachowicz \& Ogle, 2008; Connolly, 2007; McNamara, 2012).

El proceso de comprensión de textos y fluidez de lectura ha provocado fuertes discusiones y puntuales diferencias en los resultados de la investigación sobre este tema, pero no ha dejado de ser entusiasta dentro de la comunidad investigadora (Pearson \& Cervetti, 2017). La comprensión, por su propia naturaleza, es un fenómeno que sólo se puede observar indirectamente (Pearson \& Johnson, 1978), se constata cuando las personas dicen qué entendieron, de qué se sorprendieron o disfrutaron, de qué se molestaron al leer un texto. No obstante, la comprensión de un texto se puede verificar mediante la comunicabilidad de lo comprendido (Parodi, 2011) y el desempeño flexible (Perkins, 1999).

La mayoría de los estudiantes universitarios necesita adquirir habilidades que faciliten y mejoren la forma en que leen, entienden, estudian y aprenden ( $\mathrm{McNa}$ mara, 2010), sólo de esta manera mejorarán su conducta lectora y avanzarán en el nivel de comprensión de aquello que leen. Naturalmente se reflejará en su aprovechamiento académico. Consecuentemente, podrán obtener un nivel de profundidad en la comprensión de textos escritos, realizarán una lectura exitosa, alcanzando los objetivos y el dominio de conocimientos de un lector estratégico. Sin habilidades, objetivos, propósitos y conocimientos sobre el tema, los alumnos, por sí mismos, desarrollarán con gran dificultad las diversas y variadas estrategias requeridas para la comprensión.

Las investigaciones muestran que la comprensión lectora depende directamente de las estrategias que utilizan los lectores. En consecuencia, las estrategias son especialmente necesarias para aquellos estudiantes que tienen más dificultades, menos conocimientos de dominio disciplinar y bajas habilidades de lectura (Ulker, 2017). Sin embargo, Pikulski y Chard (2005) señalan que leer con éxito es una interacción compleja, puesto que implica la percepción sensorial, la memoria y la motivación. En 
resumen, leer es un proceso multifacético en el que no se debe pasar por alto la identificación de palabras o decodificación, ni la comprensión que busca la construcción del significado del texto.

La identificación y comprensión de las ideas principales de un texto escrito, necesariamente requiere de la intervención y enseñanza de estrategias de lectura apropiada de los profesores y de la práctica asidua de los propios alumnos. Se necesita, entonces, que cada institución de educación superior establezca la propia política que guíe la importancia de la alfabetización académica para que coadyuve en el proceso de comprensión de textos escritos, a fin de que los alumnos de recién ingreso a la universidad no vivan la experiencia de "forasteros" en su "comunidad disciplinar" (Carlino, 2005). Es difícil enseñar para la comprensión, pero muy necesario para superar la reproducción memorística y mecánica del conocimiento adquirido por la mayoría de los alumnos universitarios. La comprensión no se localiza en el texto escrito: nace en la mente del lector (McNamara, 2004).

\section{Lista de referencias}

Alonso, J., \& Mateos, M. (1985). Comprensión lectora: Modelos, entrenamiento y evaluación. Infancia y aprendizaje, 31-32, 5-19.

Andere, M. E. (2011). La cultura del aprendizaje: Hogar y escuela del Siglo XXI. México: Eduardo Andere Martínez.

Bachman, L. F., \& Palmer, A. S. (2009). Language Testing in Practice: Designing and Developing Useful Language Tests. Nueva York: Oxford University Press.

Blachowicz, C. L., \& Ogle, D. (2008). Reading Comprehension: Strategies for Independent Learners ( $2^{\mathbf{a}}$ ed.). Nueva York: Guilford Press.

Britton, B. K., \& Graesser, A. C. (Eds.). (1996). Models of understanding text. Nueva Jersey: Lawrence Erlbaum Associates Inc.

Broek van den, P., Virtue, S., Everson, M., Tzeng, Y., \& Sung, Y. (2002). Comprehension and memory of science texts: Inferential processes and the construction of a mental representation. En J. Otero, J. A. Leon \& A. C. Graesser (Eds.), The psychology of science text comprehension (pp. 131-154). Nueva Jersey: Erlbaum.

Carlino, P. (2005). Escribir, leer y aprender en la Universidad. Una introducción a la alfabetización académica. México: Fondo de Cultura Económica.

Cassany, D. (2010). Describir el escribir. Cómo se aprende a escribir. Barcelona: Paidós. 
Castro, D. (2013). Prueba de PISA 2009. La competencia lectora. Una explicación didáctica sobre las posibles causas del bajo nivel de desempeño de los estudiantes mexicanos en la subescala de integrar e interpretar. Tendencias pedagógicas, 21, 199-213. Recuperado de https://revistas.uam.es/tendenciaspedagogicas/ article/view $/ 2033$

Catts, H. W., \& Kamhi, A. G. (2017). Prologue: Reading Comprehension Is Not a Single Ability. Language, Speech, and Hearing Services in Schools, 48, 73-76.

Connolly, S. (2007). Successful Strategies for Reading in the Content Areas Grades 305. California: Shell Education.

Craik, F. I. M. (2002). Levels of processing: Past, Present and Future? Memory, 10(5/6), 305-318.

Craik, F. I. M., \& Lockhart, R. S. (1972). Levels of Processing: A Framework for Memory Research. Journal of Verbal Learning and Verbal Behavior, 11, 671-684.

Davoudi, M., \& Hashemi Moghadam, H. R. (2015). Critical Review of the Models of Reading Comprehension with a Focus on Situation Models. International Journal of Linguistics, 7(5), 172-187. Recuperado de http://www.macrothink. org/journal/index.php/iil/article/view/8357

Dijk van, T. (1980). Estructuras y funciones del discurso: Una introducción interdisciplinaria a la lingüística del texto y a los estudios del discurso. México: Siglo XXI.

Dijk van, T. (1992). La ciencia del texto. Barcelona: Paidós.

Fajardo, A., Hernández, J., \& González, A. (2012). Acceso léxico y comprensión lectora: un estudio con jóvenes universitarios. Revista Electrónica de Investigación Educativa, 14(2), 25-33. Recuperado de https://redie.uabc.mx/redie/article/view/305

Fletcher, J. M. (2006). Measuring Reading Comprehension. Scientific Studies of Reading, $10(3), 323-330$.

Fumagalli, J., Barreyero, J. P., \& Jaichenco, V. (2017). Niveles de fluidez lectora y comprensión de textos. Traslaciones. Revista Latinoamericana de Lectura y Escritura, 4(8), 163-186. Recuperado de http://revistas.uncu.edu.ar/ojs/index.php/ traslaciones/article/view/1063

García, N. (2017). Locales y globales. En H. Aguilar (Coord.), ¿Y ahora qué? (pp. 355369). México: Penguin Random House.

Gardner, H. (1993). La mente no escolarizada. Cómo piensan los niños y cómo deberían enseñar en las escuelas. Barcelona: Paidós.

Golder, C., \& Gaonac’h, D. (2002). Leer y comprender. Psicología de la lectura. México: Siglo XXI. 
Goldman, S. R., \& Bisanz, G. L. (2002). Toward functional analysis of scientific genres: Implications for understanding and learning processes. En J. Otero, J. A. Leon \& A. C. Graesser (Eds.), The psychology of science text comprehension (pp. 19-50). Nueva Jersey: Erlbaum.

Graesser, A. C. (2007). An Introduction to Strategic Reading Comprehension. En D. S. McNamara (Ed.), Reading Comprehension Strategies. Theories, interventions, and Technologies (pp. 3-26). Nueva York: Taylor \& Francis Group.

Graesser, A. C. (2015). Deeper learning with advances in discourse science and technology. Policy Insights from the Behavioral and Brain Sciences, 2, 42-50.

Habib, M. (2016). Assessment of Reading Comprehension. Romanian Journal for Multidimensional Education, 8(1), 125-147.

Hudson, R. F., Lane, H. B., \& Pullen, P. C. (2005). Reading fluency assessment and instruction: What, why, and how? The Reading Teacher, 58(8), $702-714$.

Hudson, R. F., Pullen, P. C., Lane, H.B., \& Torgesen, J. K. (2009). The Complex Nature of Reading Fluency: A Multidimensional View. Reading \& Writing Quarterly, 25, 4-32.

Ibáñez, R. (2012). La comprensión del discurso escrito: Una propuesta teórico-metodológica para su evaluación. Revista Signos, 45(78), 20-43.

Jenkins, J. R., Fuchs, L. S., van den Broek, P., Espin, C., \& Deno, S. L. (2003). Sources of Individual Differences in Reading Comprehension and Reading Fluency. Journal of Educational Psychology, 95, 4, 719-729.

Johnston, P. H. (1984). Reading comprehension assessment: A cognitive basis. Delaware: International Reading Association.

Kendeou, P., McMaster, K. L., \& Christ, T. J. (2016). Reading Comprehension: Core Components and Processes. Policy Insights from the Behavioral and Brain Sciences, $3(1), 62-69$.

Kerlinger, F. N., \& Lee, H. B. (2002). Investigación del comportamiento (4a ed.). México: McGraw-Hill.

Kim, Y. S., Wagner, R. K., \& Lopez, D. (2012). Developmental Relations between Reading Fluency and Reading Comprehension: A Longitudinal Study from Grade 1 to Grade 2. Journal of Experimental Child Psychology, 113(1), 93-111.

King, A. (2007). Beyond Literal Comprehension: A Strategy to Promote Deep Understanding of Text. En D. S. McNamara (Ed.), Reading Comprehension Strategies. Theories, interventions, and Technologies (pp. 267-290). Nueva York: Taylor \& Francis Group. 
Kintsch, W. (1988). The Role of Knowledge in Discourse Comprehension: A Construction-Integration Model. Psychological Review, 95(2), 163-182.

Kintsch, W. (1998). Comprehension: A Paradigm for cognition. Nueva York: Cambridge University Press.

Kintsch, W., \& Kintsch, E. (2005). Comprehension. En S. Stahl \& S. Paris (Eds.), Children's reading comprehension and assessment (pp. 71-92). Nueva Jersey: Lawrence Erlbaum Associates, Inc.

Kintsch, W., \& Rawson, K. A. (2005). Comprehension. En J. Snowling \& C. Hume (Eds.), The Science of Reading: A handbook (pp. 209-226). Massachusetts: Blackwell Publishing.

Lane, H. B., Hudson, R. F., Leite, W. L., Kosanovich, M. L., Strout, M. T., Fenty, N. S., \& Wright, T. L. (2008). Teacher knowledge about reading fluency and indicators of students' fluency growth in reading first schools. Reading \& Writing Quarterly, 25(1), 57-86. doi:10.1080/10573560802491232

Lockhart, R. S., Craik, F. I. M., \& Jacoby, L. L. (1976). Depth of processing, recognition and recall. En J. Brown (Ed.), Recall and recognition (pp. 75-102). Nueva York: Wiley.

Mansilla, V. B., \& Gardner, H. (1999). ¿Cuáles son las cualidades de la comprensión? En M. S. Wiske (Comp.), La enseñanza para la comprensión. Vinculación entre la investigación y la práctica (pp. 215-256). Buenos Aires: Paidós.

Marmolejo-Ramos, F. (mayo-agosto, 2007). Nuevos avances en el estudio científico de la comprensión de textos. Universitas Psychologica, 6(2), 331-343.

McNamara, D. S. (2004). Aprender del texto: Efectos de la estructura textual y de las estrategias del lector. Revista Signos, 37(55), 19-30.

McNamara, D. S. (2010). Strategies to read and learn: overcoming learning by consumption. Medical Education, 340-346.

McNamara, D. S. (2012). Reading Comprehension Strategies: Theories, Inventions, and Technologies. Nueva Jersey: Psychology Press.

McNamara, D. S., \& Magliano, J. (2009). Toward a Comprehensive Model of Comprehension. En B. Ross (Ed.), The Psychology of Learning and Motivation (vol. 51, pp. 297-384). Burlington: Academic Press.

Miras, M., Solé, I., \& Castells, N. (abril-junio, 2013). Creencias sobre lectura y escritura, producción de síntesis escritas y resultados de aprendizaje. Revista Mexicana de Investigación Educativa, 18(57), 437-459. Recuperado de https://www. comie.org.mx/revista/v2018/rmie/index.php/nrmie/article/view/253 
Oostendorp van, H., \& Goldman, S. R. (Eds.). (1999). The construction of mental representations during reading. Nueva Jersey: Lawrence Erlbaum Associates.

Organización para la Cooperación y el Desarrollo Económicos. (2016). PISA 2015 results: Excellence and equity in education. París: Autor.

Parodi, G. (2011). La Teoría de la Comunicabilidad: Notas para una concepción integral de la comprensión de textos escritos. Revista Signos, 44(76), 145-167.

Pearson, P. D., \& Cervetti, G. N. (2017). The Roots of Reading Comprehension Instruction. En S. E. Israel (Ed.), Handbook of Research on Reading comprehension (pp. 12-56). Nueva York: The Guilford Press.

Pearson, P. D., \& Hamm, D. N. (2005). The assessment of reading comprehension: a review of practices -past, present, and future. En S. G. Paris \& S. A. Stahl (Eds.), Children's reading comprehension and assessment (pp. 13-69). Nueva Jersey: Lawrence Erlbaum Associates, Inc.

Pearson, P. D., \& Johnson, D. D. (1978). Teaching reading comprehension. Nueva York: Holt, Rinehart y Winston.

Pérez, M. J. (2005). Evaluación de la comprensión lectora: dificultades y limitaciones. Revista de Educación, 121-138. Recuperado de http://www.revistaeducacion. mec.es/re2005_08.htm

Perfetti, C., Landi, N., \& Oakhill, J. (2005). The acquisition of reading comprehension skill. En M. J. Snowling \& C. Hulme (Eds.), The science of reading: A handbook (pp. 227-247). Oxford: Blackwell.

Perfetti, C., \& Stafura, J. (2014). Word Knowledge in a Theory of Reading Comprehension. Scientific Studies of Reading, 18, 22-37.

Perkins, D. (1999). ¿Qué es la comprensión? En M. S. Wiske (Comp.), La enseñanza para la comprensión. Vinculación entre la investigación y la práctica (pp. 68-92). Argentina: Paidós.

Pikulski, J. J., \& Chard, D. J. (marzo, 2005). Fluency: Bridge between deeoding and reading comprehension. The Reading Teacher, 58(6), 510-519.

Rupp, A. A., Ferne, T., \& Choi, H. (2006). How assessing reading comprehension with multiple-choice questions shapes the construct: a cognitive processing perspective. Language Testing, 23(4) 441-474.

Sánchez, E. (2014). Comprensión lectora. En R. O. González (Coord.), Habilidades lingüísticas de los estudiantes de primer ingreso a las Instituciones de Educación Superior (pp. 170-183). México: Asociación Nacional de Universidades e Instituciones de Educación Superior. 
Schmitt, N., Jiang, X., \& Grabe, W. (2011). The Percentage of Words Known in a Text and Reading Comprehension. The Modern Language Journal, 95(1), 26-43.

Shahnazari, M. T., \& Dabaghi, A. (2014). A Critical Overview of Models of Reading Comprehension with a Focus on Cognitive Aspects. IJREL, 2(1), 7-19.

Snow, C. E. (2010). Reading Comprehension: Reading for Learning. International Encyclopedia of Education, 5, 413-418.

Stevens, S. S. (junio, 1946). On the Theory of Scales of Measurement. Science, $103(2684), 677-680$.

Stevens, S. S. (agosto, 1968). Measurement, Statistics, and the Schemapiric View. Science, $161(3844), 849-856$.

Tijero, N. T. (2009). Representaciones mentales: discusión crítica del modelo de situación de Kintsch. Onomazein, 19(1), 111-138.

Ulker, U. (diciembre, 2017). Reading Comprehension Strategies. International Journal of Social Sciences \& Educational Studies, 4(3). 140-145.

Wiske, M. S. (1999). La enseñanza para la comprensión. Vinculación entre la investigación y la práctica. Buenos Aires: Paidós.

Yildirim, K., Rasinski, T., \& Kaya, D. (2017). Fluency and Comprehension of Expository Texts in Turkish Students in Grades Four through Eight. Education and Science. 1-12.

Yildirim, K., Rasinski, T., \& Kaya, D. (2018). Fluency and comprehension of narrative texts in Turkish students in grades 4 through 8. Education 3-13, 1-10.

Yildiz, M., \& Çetinkaya, E. (2017). The Relationship between Good Readers' Attention, Reading Fluency and Reading Comprehension. Universal Journal of Educational Research, 5(3), 366-371. 